\title{
Spectrum of Mammography and Ultrasonography Findings in Imaging of Post-Surgical Breast: Many Facets!
}

\author{
Surabhi Mehrotra ${ }^{1}$, Anjum Syed ${ }^{2}$, Bina Ravi ${ }^{3}$, Aakriti Kapoor ${ }^{4}$, Divya Pandey ${ }^{5}$, Priyanka Gupta ${ }^{6}$, Prateek Sharda ${ }^{7}$ \\ ${ }^{1}$ Senior Resident, PDCC Fellow, Department of IBCC, ${ }^{2}$ Additional Professor, Department of IBCC, ${ }^{3}$ Senior Professor, Department \\ of IBCC, ${ }^{4}$ Senior Resident, PDCC Fellow, Department of IBCC, ${ }^{5}$ Senior Resident, PDCC Fellow, Department of IBCC, ${ }^{6}$ Senior \\ Resident, PDCC Fellow, Department of IBCC, ${ }^{6}$ Senior Resident, Department of IBCC, All India Institute of Medical Sciences, \\ Rishikesh, Uttarakhand, India
}

Corresponding author: Dr Surabhi Mehrotra, Senior Resident, Department of IBCC, Breast Clinic, AlIMS Rishikesh, India

DOI: http://dx.doi.org/10.21276/ijcmsr.2019.4.3.44

How to cite this article: Surabhi Mehrotra, Anjum Syed, Bina Ravi, Aakriti Kapoor, Divya Pandey, Priyanka Gupta, Prateek Sharda. Spectrum of mammography and ultrasonography findings in imaging of post-surgical breast: many facets!. International Journal of Contemporary Medicine Surgery and Radiology. 2019;4(3):C200C206.

\section{A B S T R A C T}

Introduction: Postoperative breast surgery findings include skin thickening, increased focal breast tissue density, architectural distortion, seroma or fluid/ air collection in the early few months, which decrease over a period of time. Follow up imaging findings include parenchymal scarring, skin thickening / deformity, areas of dystrophic calcification/ fat necrosis and recurrent mass lesions at the post surgical site. The purpose of the study was to evaluate Mammographic and ultrasonographic (USG) findings in the patients who have undergone breast surgery.

Material and methods: A total of 50 patients with history of breast carcinoma surgery were evaluated in the Integrated breast care centre (IBCC) at All India Institute of Medical Sciences, Rishikesh, Uttarakhand (U.K.), India. Mammographic and ultrasonographic evaluation of breast and axilla were done.

Results: Ultrasonography and mammography findings in post surgery patients were illustrated and described. Timing of the appearance of imaging findings were categorized according to the time of presentation.

Conclusion: Post surgical changes should be thoroughly evaluated. Ultrasonography and mammography are the mainstay in the follow up of patients along with clinical examination. Comparison with prior imaging place an important role in reaching to a radiological diagnosis.

Keywords: Post-Operative, Ultrasonography, Mammography

\section{INTRODUCTION}

Normal post operative findings on mammography include skin thickening, architectural distortion, and increased density in more than $50 \%$ of patients in the initial few months of surgery. Only anecdotal examples of postoperative changes have been reported. ${ }^{1,2}$ The findings reduce in intensity over time and get stable on follow up mammograms in approximately 3 to 5 years time. On ultrasound, the immediate post operative site shows fluid collectionsseromas or hematomas, skin thickening and edematous changes. Seromas can be completely anechoic or may contain low level internal echoes, internal septas or dependent debris within. Surgical clips are seen at the site of post surgical site. In some of the cases, recurrent mass lesions can be seen at the site of previous surgeries.

The purpose of the study was to evaluate Mammographic and ultrasonographic (USG) findings in the patients who have undergone breast surgery for carcinoma, either breast conserving or modified radical mastectomy and to provide a pictorial review of post surgical breast based on mammographic and USG findings.

\section{MATERIAL AND METHODS}

From the period of January 2019 to June 2019, a total of 50 patients (all females) with history of breast carcinoma surgery (6 months to 5 years interval) who presented for clinical evaluation in the Integrated Breast Care Centre (IBCC), tertiary centre at All India Institute of Medical Sciences, Rishikesh, Uttarakhand (U.K.), India were recruited for dedicated mammographic and ultrasonographic evaluation of breast and axilla. Post operative findings in breast were correlated with clinical features and histopathology findings where applicable.

Inclusion Criteria: All patients who had undergone some form of breast surgery including tumour excisional biopsy (done outside),simple lumpectomy, breast conserving surgery, quadrantectomy, modified radical mastectomy, total mastectomy, radical mastectomy, extended radical mastectomy, axillary dissection; in 0-5 years interval were included in our study. Study also included breast implant case. Mammograms including Full-filed digital mammogram (FFDM) and tomosynthesis were done on Hologic Selenia G-XR-62728 including - Craniocaudal (CC) and 
Mediolateral oblique (MLO) views. Additional mediolateral (ML) or exaggerated views were taken whenever indicated. USG were done using Mindray Z-6 machine using high frequency transducer $(5-10 \mathrm{Mhz})$.

\section{RESULTS}

Out of the 50 patients, 35 (70\%) patients had skin thickening at the post operative site (Table 1, fig-1). Out of these 35 patients, 32 (64\%) patients have seroma underlying the postoperative site. 18 (36\%) patients out of 50 had post operative architectural distortion and increased breast tissue density at the post operative site. $10(20 \%)$ patients presented with recurrent mass lesion at the same site. $10(20 \%)$ out of 50 patients had skin-fold. 9 (18\%) patients had presented with fat necrosis and dystrophic calcification. 4(8\%) patients presented with oil cysts. Only 1 (2\%) patient was post breast reconstruction surgery with fat grafting and $1(2 \%)$ patient

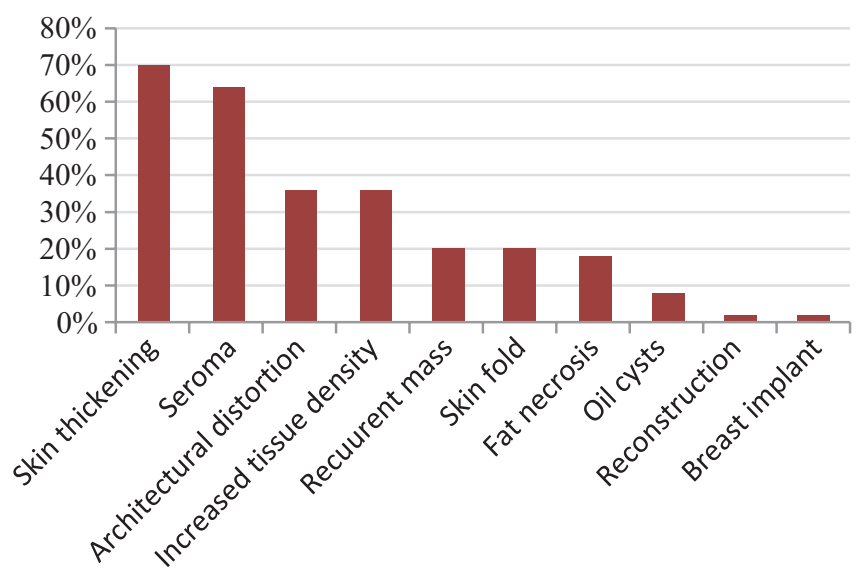

Figure-1: Percentage of Post operative findings

Age (in years)

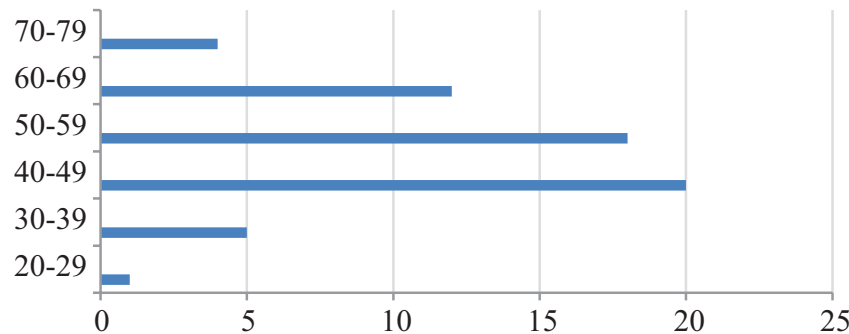

Figure-2: Distribution of patients according to age (in years)

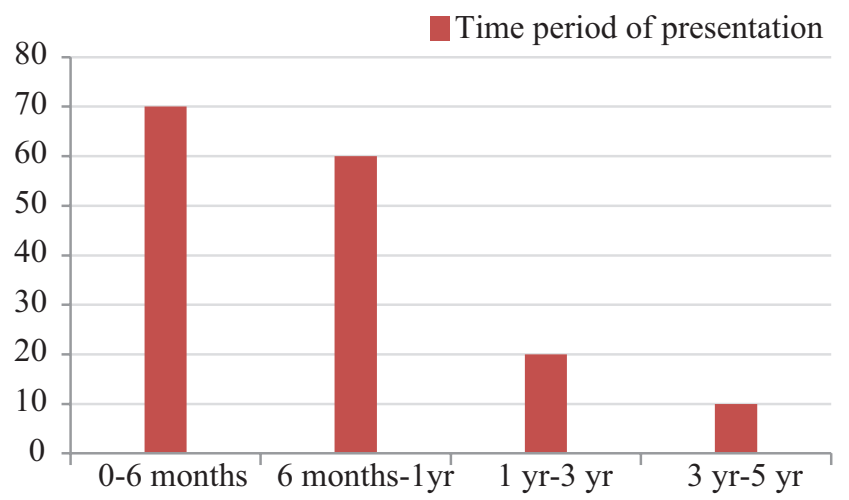

Figure-3: Distribution of patients according to time of presentation

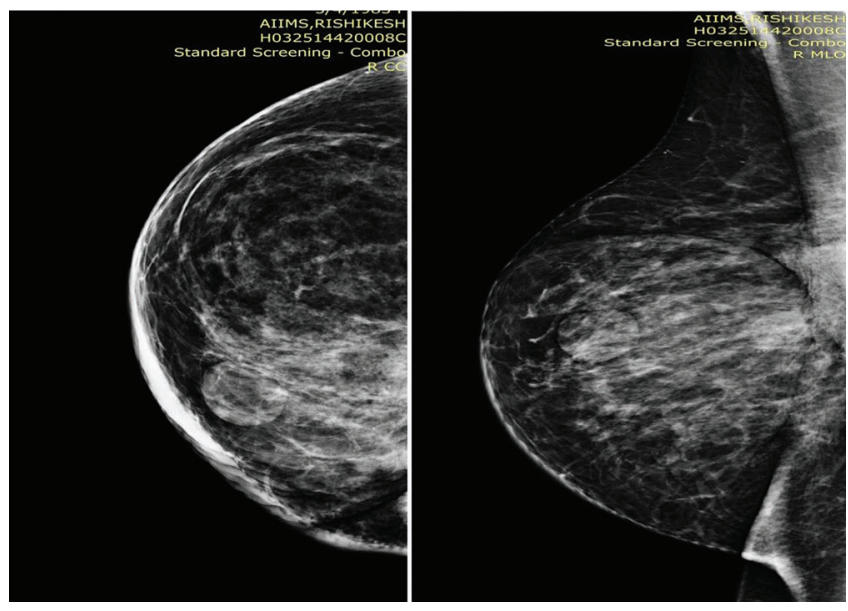

Figure-4a and 4b: Mammogram of a 35 year female with post lumpectomy status showing a curvilinear scar running from $11-5$ o' clock positions with overlying skin thickening.
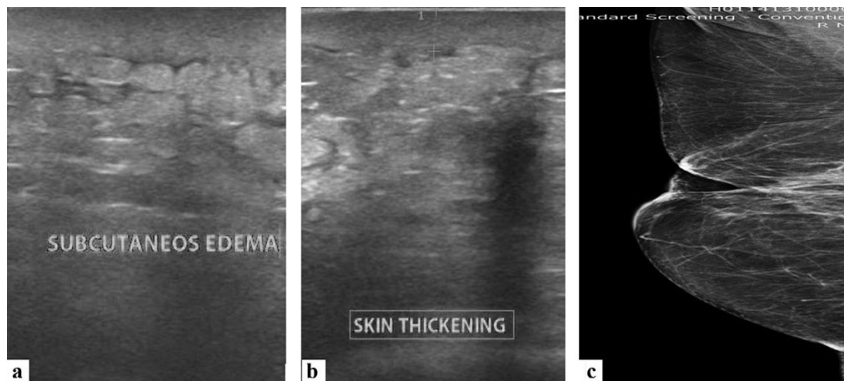

Figure-5:Ultrasonography (a and b)showing skin thickening along with subcutaneous edema seen within the fat lobules. Mammogram (c) of a 64 years female with history of quadrant lumpectomy for ductal carcinoma in situ, post chemotherapy and radiotherapy status done 4 years ago- showing focal skin fold and skin thickening at the scar site.

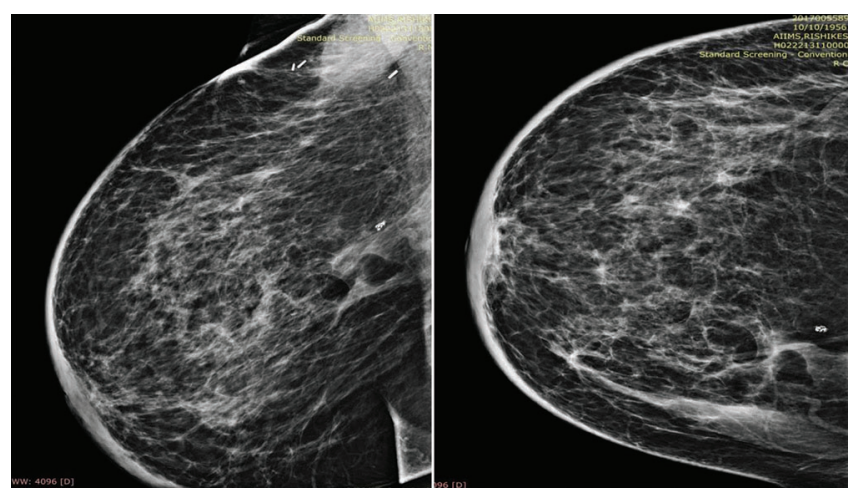

Figure-6 and 6b: Mammogram of a 61 year old female who underwent breast conservation surgery 2 years ago for right breast intraductal carcinoma, post chemotherapy/ and radiotherapy status showing few well defined lobulated low density lesions seen in upper inner quadrant showing a foci of macrocalcification posteriorly. Another ill-defined area of high density in upper inner quadrant, posteriorly in retro-mammary region-suggestive of seromas (which was confirmed on aspiration and cytology). Diffuse skin and trabecular thickening seen.Right axilla and upper outer quadrant shows multiple linear high density areas- surgical clips 


\begin{tabular}{|l|c|c|c|}
\hline Imaging feature & Number of patients & \% of patients & Time of presentation \\
\hline Skin thickening & 35 out of 50 patients & $70 \%$ & $0-6$ months \\
\hline Seroma & 32 out of 50 patients & $64 \%$ & $0-6$ months \\
\hline Architectural distortion & 18 out of 50 patients & $36 \%$ & $0-6$ months \\
\hline Increased tissue density & 18 out of 50 patients & $36 \%$ & $0-6$ months \\
\hline Recurrent mass & 10 out of 50 patients & $20 \%$ & 6 months-1 year \\
\hline Skin fold & 10 out of 50 patients & $20 \%$ & $1-3$ year \\
\hline Fat necrosis, dystrophic calcification & 9 out of 50 patients & $18 \%$ & $1-3$ year \\
\hline Oil cysts & 4 out of 50 patients & $8 \%$ & 6 months- 1 year \\
\hline Reconstruction & 1 out of 50 patients & $2 \%$ & $1-7$ year \\
\hline Breast implant & 1 out of 50 patients & $2 \%$ & \\
\hline \multicolumn{2}{|l|}{ Table-1: Percentage of Post operative findings and most common observed time of presentation } \\
\hline
\end{tabular}

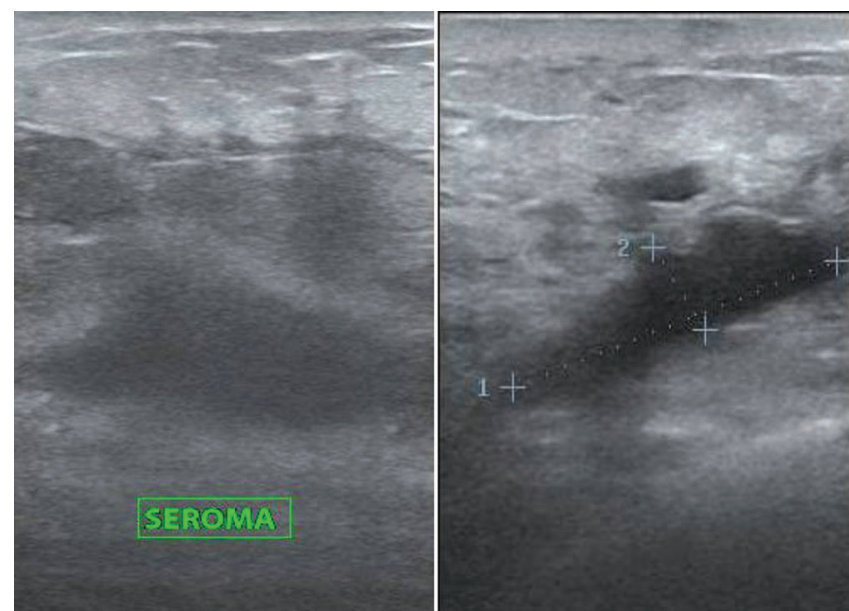

Figure-7a and 7b: Ultrasonography showing an ill defined hypoechoic collection beneath the scar site with surrounding increased echogenicity and inflammed fat.

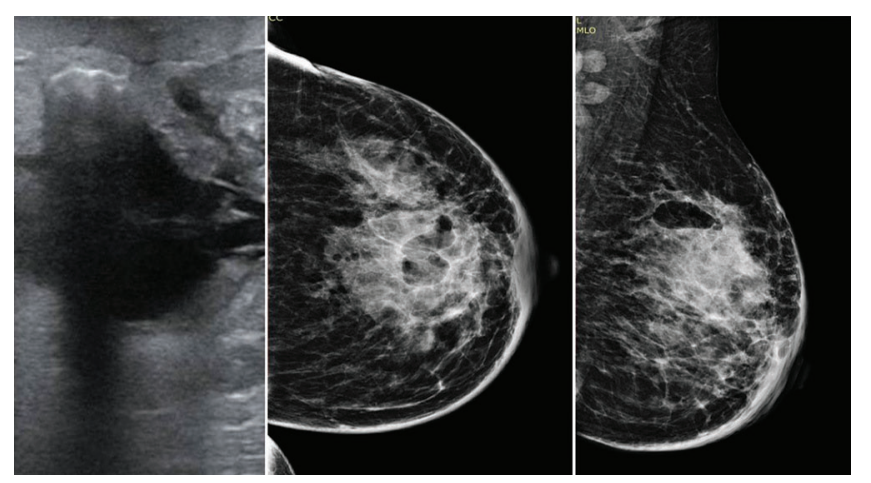

Figure-8a, 8b and 8c: Ultrasonography of a 35 year old female with undocumented lumpectomy done outside in a private centre showing a loculated heterogeneously hypoechoic collection with posterior acoustic enhancement and multiple septa, internal echoes and echogenic areas casting dirty shadow due to air within seroma and increased echogenicity of surrounding breast parenchyma. Mammogram of the same patient (craniocaudal and mediolateral oblique view) showing an irregular high density area in upper central and inner quadrant showing multiple lucent areas-likely air. Findings are suggestive of Seroma with air-post operative changes. The patient underwent biopsy and was proven malignancy on histopathology.
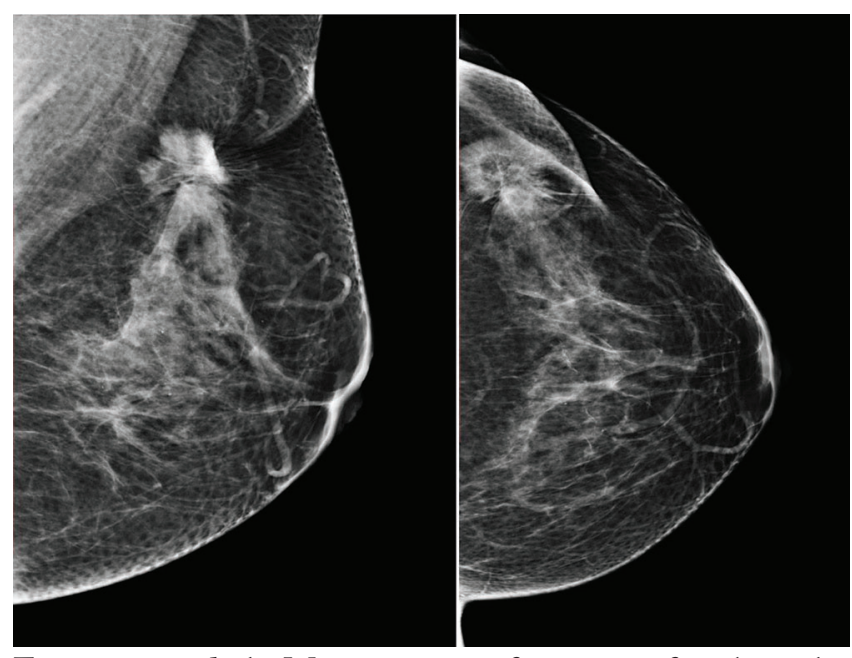

Figure-9a and 9b: Mammogram of a 44 year female s who underwent breast conservation surgery 8 months ago for invasive ductal carcinoma left breast shows architectural distortion with central lucency in lateral and upper quadrant at lumpectomy site.
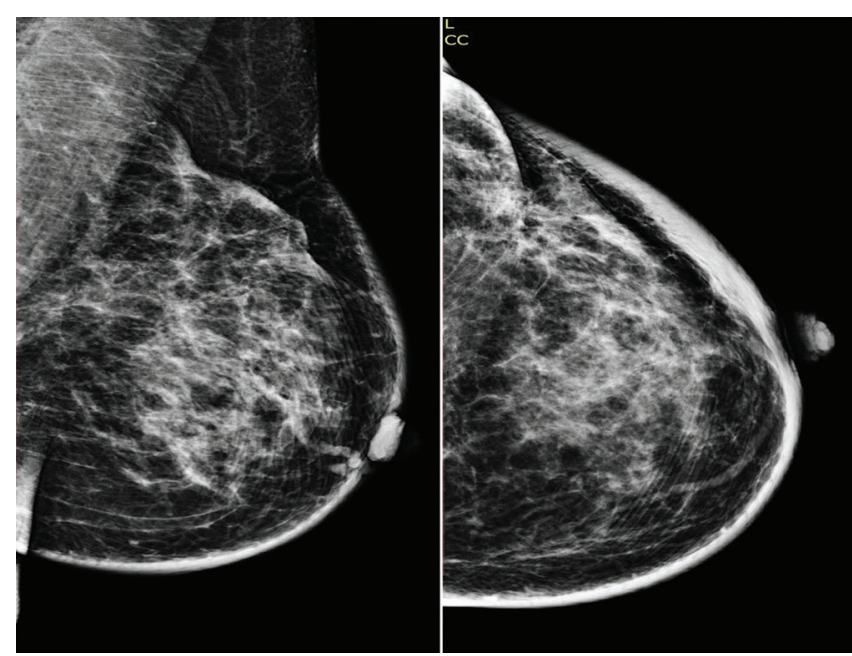

Figure-10a and 10b: Mammogram of a 35 year old female who underwent left breast conversation surgery for carcinoma left breast 1.2 years back - showing architectural distortion with diffuse skin thickening in the left upper outer quadrant at the operative site with few areas of lucency suggestive of fat necrosis. 

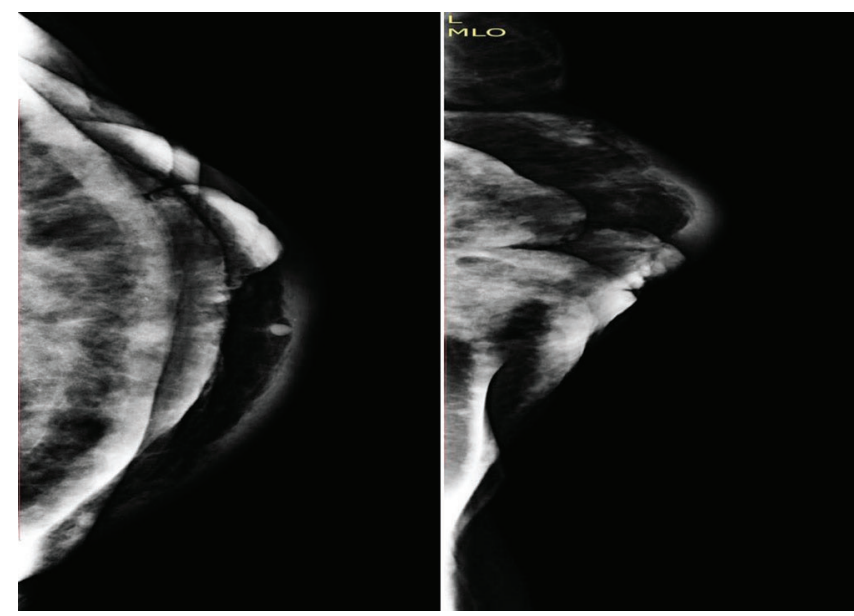

Figure-11a and 11b: Mammogram Craniocaudal (a)and mediolateral oblique (b)view in a 25 year old with quadrant lumpectomy done 2 years back showing - markedly reduced breast tissue with rest of the breast tissue showing architectural distortion with irregularity and clustered fine pleomorphic calcification with overlying skin thickening and retraction- recurrent lesion at the same site.
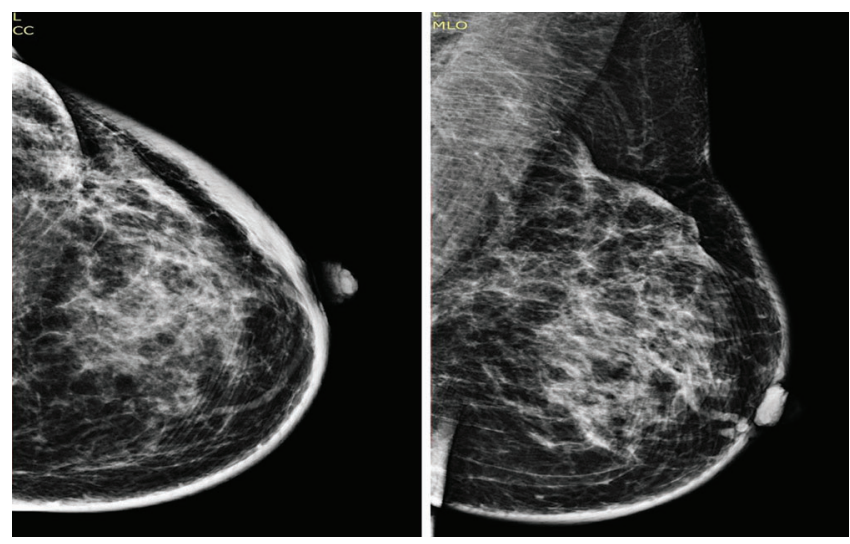

Figure-12a and 12b: Mammogram craniocaudal (a) and mediolateral view(b) of a 36 year old female in post breast conservation surgery done 1 year back showing a well defined iso to high density area with surrounding peripheral lucency seen in upper outer quadrant. Similar few low density areas seen scattered in inner quadrant - fat necrosis.

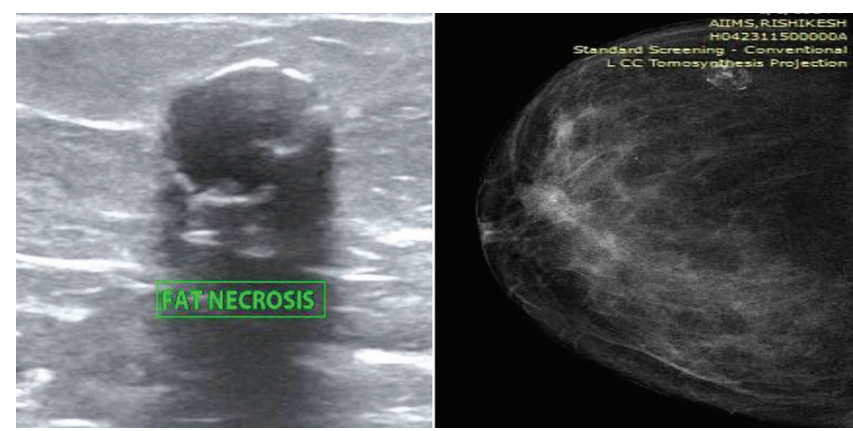

Figure-13a and 13b: Ultrasonography of the breast reveals a well-defined oval hypoechoic lesion showing peripheral hyperechoic rim and eccentric hyperechoic component casting posterior acoustic shadowing in subcutaneous plane. Mammogram of the same patient reveals a lucent lesion with discontinuous rim calcification and coarse macrocalcifications in upper inner quadrant - suggestive fat necrosis.

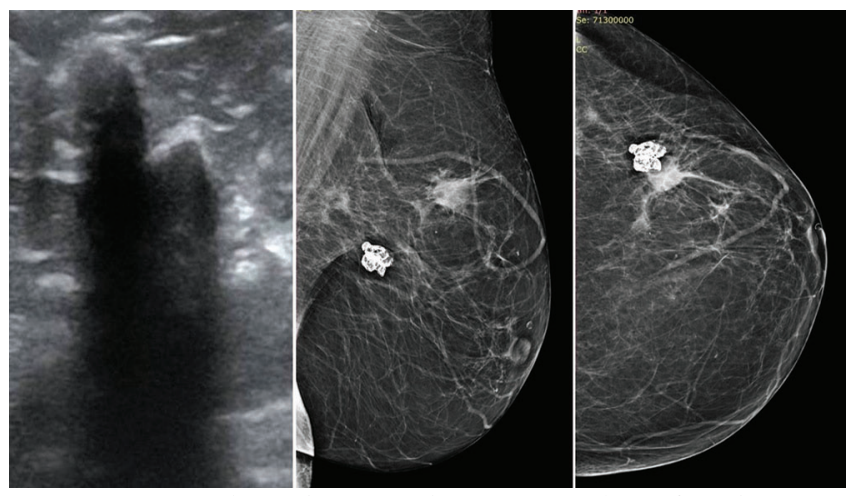

Figure-14a, 14b and 14c: Ultrasonography of a 57 years old female with post lumpectomy status left breast showing an ill-defined area showing multiple foci of coarse macrocalcification in upper outer quadrant beneath the scar site. Mammogram mediolateral oblique and cranio-caudal view of the same patient showing a coarse ill-defined area of macro-calcification - suggestive of dystrophic calcification.

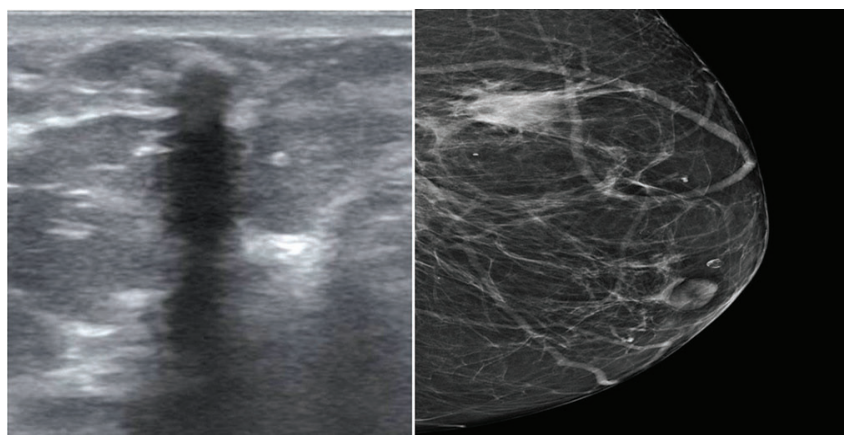

Figure-15a and 15b: Ultrasonography of a 57 year old female with post lumpectomy status showing a small well defined hypechoic lesion with strong posterior acoustic enhancement. Mammogram craniocaudal view of the same patient showing a well defined lucent lesion with rim calcification in retroareolar region of breast - suggestive of oil cyst.
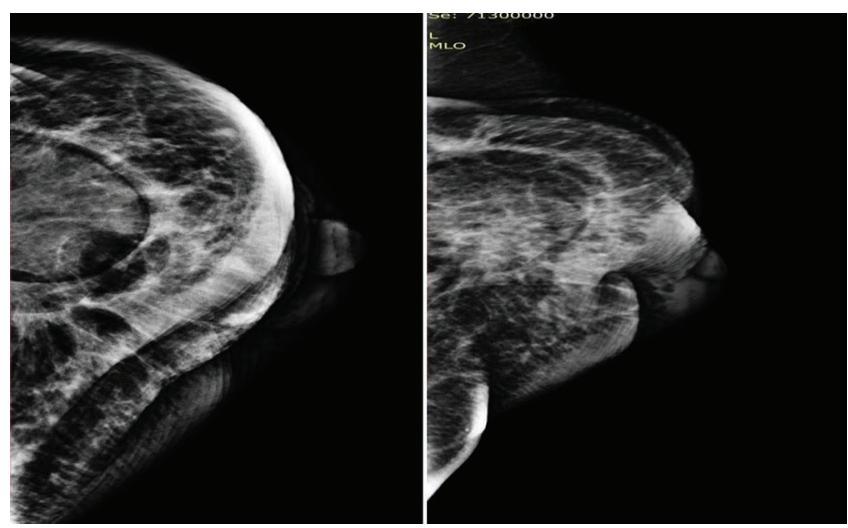

Figure-16a and 16b: Mammogram craniocaudal view of a 47 year old female with Post operative status of left skin and nipple sparing mastectomy with fat grafting showing architectural distortion in entirety with multiple folds of retracted skin with skin thickening. A well-defined iso to high density area with surrounding peripheral lucency seen in upper outer quadrant. Similar few low density areas seen scattered in inner quadrant - post operative changes with fat necrosis. 

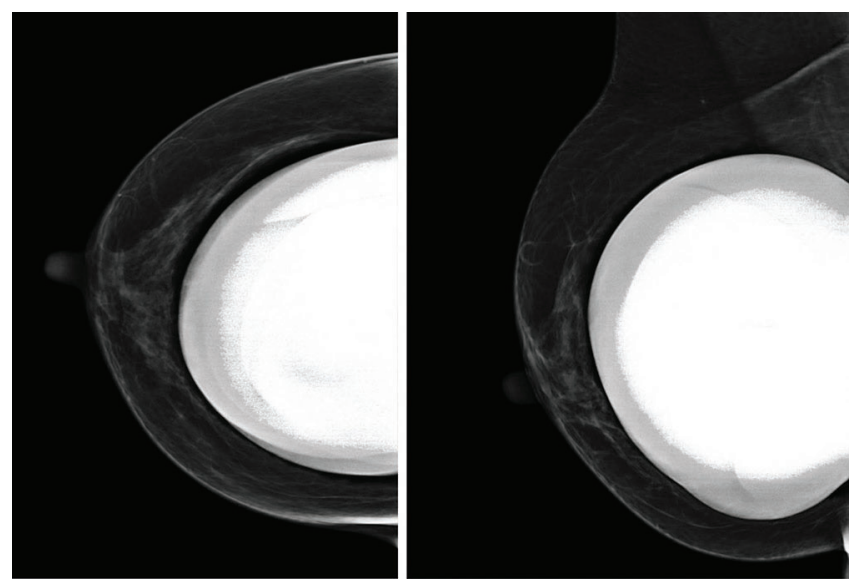

Figure-17a and17b: Mammogram craniocaudal and mediolateral view of right breast a 60 year old female with bilateral subglandular breast implants, 7 years back, presented with complains of left breast pain since 4 months. Normal silicone implant with maintained outline. No evidence of any implant rupture seen.

Note: Subsequent MRI (not shown) was done for implant integrity which showed intracapsular folds.

was post breast implant surgery. Mean age group of the patients was ranging between 40-60 years which constituted $76 \%$ of the total patients (fig 2). Out of the total 50 patients, 15(30\%) patients had simple lumpectomy, 20(40\%) had undergone breast conservation surgery, $2(4 \%)$ had undergone tumour excisional biopsy, $8(16 \%)$ had modified radical mastectomy and 5(10\%) were post total mastectomy patients. $4(8 \%)$ patients had undergone biopsy and 7(14\%) had undergone Fine needle aspiration cytology (FNAC) in our study group for evaluation. 35(70\%)patients were evaluated in 0-6 months after surgery, 30 (60\%) patients in 6 months- 1 year, $10(20 \%)$ patients in a period of $1-3$ years and $5(10 \%)$ patients presented 3-5 years after surgery (fig 3). Patients were called for every 6 monthly follow up and then annually.

\section{DISCUSSION}

\section{Skin thickening, skin fold and Surgical scar}

Normal post operative findings include architectural distortion, increased density and parenchymal scarring in at least $50 \%$ patients. These findings decrease in severity over time. These findings get stable in 3-5 years on subsequent mammograms. Scar appears as an area of chronic architectural distortion or a spiculated mass more evident on one projection than the other. Normal skin thickness is less than $2 \mathrm{~mm}$, however in post breast conservation surgery patients, thickness can be $10 \mathrm{~mm}$ or more. Any recent change in edema or skin thickening should raise the suspicion of recurrence and should be closely evaluated. By 2 to 3 years after breast conservation therapy, the skin has returned to near-normal thickness, although mild thickening persists in approximately $30 \%$ of the patients. ${ }^{3}$ Nearly all patients who have had axillary dissection or radiation therapy have breast edema. ${ }^{4,5}$ Skin and trabecular thickening can also be seen secondary to radiation therapy ${ }^{6}$ (figure-4,5).

\section{Seroma}

Asymmetric soft-tissue densities are expected at postoperative sites. ${ }^{7}$ They have been described as being more common in older and obese patients. ${ }^{11} \mathrm{MRM}$ is more frequently associated with seroma formation than lumpectomy. ${ }^{8-12}$ Postsurgical seroma after MRM occurs in $20-50 \%$ of patients compared with $9-20 \%$ of patients after lumpectomy. ${ }^{8-13}$ In rare cases, when a mammogram is taken within few days of surgery, a round or oval mass in the post operative site is seen representing a seroma or hematoma. Air can be present or absent within it. This mass represents the biopsy-cavity which is filled with fluid that should resolve over time. The adjacent breast tissue shows thickening of trabeculae and increased density in subcutaneous fat caused by local edema and hemorrhage. Over the subsequent weeks, the post-operative site resorbs the air and fluid collection and the collection is replaced by fibrosis, scarring and residual focal skin thickening. On ultrasonography, these are seen as anechoic fluid collections. Later on, thickened nodular margins may develop with internal echoes within. A hematoma is a less common postsurgical complication, with a reported incidence of $2-10 \%$ of breast cancer surgery cases. ${ }^{9}$ Hematomas are slightly more dense than seromas on mammograms due to hemosiderin (fig-6,7,8).

\section{Architectural distortion}

Distortion of breast parenchyma is commonly seen in post operative patients after breast conservation surgery. On mammogram, this is seen as areas of increased trabecular thickening, increased density, area of focal skin retraction, spiculations seen radiating into the breast parenchyma. Architectural distortion is often a mimic of recurrence in breast carcinoma. Non association with mass, variable appearances on different projections and presence of interspersed areas of fat goes in favour of architectural distortion. Comparison with prior imaging is invaluable in excluding malignancy. On ultrasonography, architectural distortion is seen as area of altered echogenicity with distortion of adjacent cooper's ligaments (fig-9,10).

\section{Recurrence}

Recurrent breast cancer is the development of malignancy within the same breast either at the same site or close to the previously operated site in a period of more than two years following surgical excision. The rate of local recurrence may be as high as $19 \%$ in 10 years. Maximum cases in recurrence in breast occurs 4-6 years post treatment (fig 11). Tumor recurrence varies from $6 \%$ to $10 \%$, at rates reported as $1 \%$ to $2 \%$ or more per year after treatment. ${ }^{14,15,16,17,18}$

\section{Fat necrosis}

Fat necrosis is a benign (non-cancerous) breast condition that happens when an area of the fatty breast tissue is damaged, usually as a result of injury to the breast. It can also happen after breast surgery or radiation treatment. It can take months to years to develop. Sometimes, inflammation may lead to fibrotic response and can produce appearance similar to desmoplastic reaction in breast along with calcium deposition which can mimic appearance of cancer. Lipolysis, inflammatory cell infiltration, and hemorrhage occur acutely followed by the formation of fibrous scar or a calcified cystic mass as the lesion evolves ${ }^{19}$, resulting in a variable imaging appearance. On ultrasonography, seen as hypoechoic mass 
with well defined margins and mural nodules within. On Mammography, fat necrosis is seen as an encaspsulated fat containing lesion or large calcifications in the surgical bed. Initially it can be seen as ill-defined, irregular area with spiculations (fig -12,13).

\section{Dystrophic calcification}

Morphologically these calcifications are large (> $5 \mathrm{~mm}$ ) and irregular in outline with central lucencies, with no associated mass/density and always occur at the site of surgery. ${ }^{20}$ These are manifestation of fat necrosis. A dystrophic calcification have smooth margins, unlike the irregular margins seen in microcalcification suggestive of malignancy. They are common after surgery and radiotherapy. ${ }^{21}$ On mamography, the presence of radiolucent area within is indicative of existence of fat (fig 14).

\section{Post operative oil cyst}

Oil cyst is a form of fat necrosis. The damaged body tissue is repaired and is replaced by firm scar tissue. The classic appearance of fat necrosis in the breast is a radiolucency that is visible due to its thin capsule. These have a variable appearance on ultrasound but may be hypoechoic. They are well-circumscribed hypoechoic lesions with variable through-transmission of sound. On mammography, these are seen as radiolucent rounded lesions with fat density in the centre. Peripheral calcification if present is seen as eggshell calcification (fig 15).

\section{Reconstruction}

Mammographic findings in the patients undergoing breast reconstruction includes changes in the parenchymal architecture, cranial or caudal displacement of the nipple, development of patchy densities due to tissue removal and scarring and also fat necrosis. A new baseline mammogram should be obtained 6 months after surgery. After a mastectomy, breast reconstruction can be done by means of autogenous tissue transfer or implants. The most common autogenous tissue transfer site is from the panniculus or from a free myocutaneous flap. The most frequent location of the donor tissue is from a flap harvested from the latissimus dorsi muscle or the transverse rectus abdominis muscle (TRAM) flap. Ultrasonographic and mammographic findings are related to the development of dystrophic changes within the donor tissue, such as oil cyst, fat necrosis and dystrophic calcification. Sometimes tissue scarring also is a common mimic of cancer, which can lead to warrant of biopsy in those cases (fig 16).

\section{Breast Implant}

An increasing number of patients have breast implants for cosmetic augmentation of the breast, reconstruction after mastectomy or correction of congenital malformations. ${ }^{22}$ Breast implants may be placed in a subglandular (anterior to the pectoralis major muscle) or subpectoral (posterior to the pectoralis major muscle) location. The technique used for mammography in breast implant is Eklund technique. Only 1 patient of implant was included in our study. Extrasilicone ruptures can be easily indentified while intrasilicone ruptures are hard to identify on mammogram. On mammography, implants appear as oval masses with a dense silicone envelope in outer aspect and a less radio-opaque center. Normal membrane foldings and glandular tissue can be seen through the implant depending on the penetration used. Ultrasound is helpful in the assessment of contour, content and morphology of implant. The normal undulations are seen as wavy echogenic lines with or without intervening fluid. Both the silicone and saline implants appear anechoic surrounded by linear echogenic envelope (fig 17).

\section{CONCLUSION}

The post surgical changes in breast need clinical correlation and proper evaluation with the clinical history. Skin thickening, edema, increased breast density and increased trabecular thickening are the findings commonly seen in post operative patients. Any new appearance of architectural distortion or mass lesion or newer microcalcifications should raise suspicion for development of a new cancerous mass lesion and should be thoroughly evaluated. Stability is defined as no interval change on two successive mammographic studies ${ }^{23}$ and is generally observed at around 2-3 years after the completion of radiation therapy. Fat necrosis is often a mimic of breast cancer in post operative patients. Such patients should be kept in close follow -up and fully evaluated if clinically appears to be suspicious. Inconclusive diagnosis on ultrasonography and mammography can lead to biopsy in such patients. Thorough knowledge of the imaging features in post operative breast is essential in decision making and follow up.

\section{REFERENCES}

1. Stigers, K., King, J., Davey, D. and Stelling, C. Abnormalities of the breast caused by biopsy: spectrum of mammographic findings. American Journal of Roentgenology 1991;156(2):287-291.

2. Peters ME. Fagerholm M1. Scanlan KA. et al. Mammographic evaluation of the postsurgical and imidiated breast. 1988:8(3):873-899

3. Libshitz HI, Montague ED, Paulu s DD: Skin thickness in the therapeutically irradiated breast. AJR Am J Roentgenol 1978;130 (5):345-347.

4. Bland KI, Copeland EM Ill: General principles of mastectomy. In Bland KJ, Copeland EM III (eds): The Breast. Philadelphia, WB Saunders, 1991, p 570 book chapter.

5. Harris JR, Recht A: Conservative surgery and radiotherapy. 111 Harris JR, Hellina S, Henderson IC, et a 1 (eds): Breast Disease, ed 2. Philadelphia, JB Lippincott, 1991, p 395

6. Krishnamurthy R, Whitman GJ, Stelling CB, Kushwaha AC. Mammographic findings after breast conservation therapy. RadioGraphics 1999; 19 (1):S53-S62

7. Stigers KB, King JG, Davey DO et al. Abnormalities of the breast caused by biopsy: Spectrum of mammographic findings. AJR Am J Roentgenol 1991;156 2):287291.

8. Gonzalez EA, Saltzstein EC, Riedner CS, Nelson BK. Seroma formation following breast cancer surgery. Breast J 2003; 9 (4):385-388

9. Vitug AF, Newman LA. Complications in breast surgery. Surg Clin North Am 2007; 87 (6):431-451 
10. Pogson CJ, Adwani A, Ebbs SR. Seroma following breast cancer surgery. Eur J SurgOncol 2003; 29 (1):711-717

11. Zieliński J, Jaworski R, Irga N, Kruszewski JW, Jaskiewicz J. Analysis of selected factors influencing seroma formation in breast cancer patients undergoing mastectomy. Arch Med Sci. 2013;9 (3):86-92

12. Hashemi E, Kaviani A, Najafi M, Ebrahimi M, Hooshmand H, Montazeri A. Seroma formation after surgery for breast cancer. World J Surg Oncol 2004;9 (1):44.

13. Woodworth PA, McBoyle MF, Helmer SD, Beamer RL. Seroma formation after breast cancer surgery: incidence and predicting factors. Am Surg 2000; 66 (3):444-450

14. Barr LC, Brunt AM, Goodman AG, et al: Cncontrolledloca 1 recurrence after treatment of breast cancer with breast conservation. Cancer 1989;64 (5):12031207.

15. Dubois JB, Saumon-Reme M, Gary-Bobo J, et al: Tumorectomy and radiation therapy in early breast cancer: A report on 392 patients. Radiology 1990;175 (1):867871.

16. Fisher B, Bauer M, Margolese R, et al: Five year results of a transabdomized clinical trial comparing total mastectomy and segmental mastectomy with and without radiation in the treatment of breast cancer. 1 iEngl J Med 1985;312 (6):665-673.

17. Haffty BG, Goldberg NB, Rose M, et al: Conservative surgery with radiation therapy in clinical stage I and II breast cancer. Arch Surg 1989;124 (2):1266-1270.

18. Stehlin is, delpolyi PO, Greeff $\mathrm{Pi}$, et al: A ten year study of partial mastectomy for ca rcinoma of the breast. Surg Gynecol Obstet 1987;165 (4):191-198.

19. Rosen PP. Inflammatory and reactive tumors. Chapter In: Rosen PP. Rosen's Breast Pathology, 3rd ed. Philadelphia, PA: Lippincott, Williams, \& Wilkins, 2009:33

20. Gutierrez R, Horst KC, Dirbas FM, Ikeda DM. Breast imaging following breast conservation therapy. In: Dirbas FM and Scott-Conner CEH editor. Breast surgical techniques and inter disciplinary management. New York: Springer Science Business Media; 2011. pp. 975-995.

21. D’Orsi CJ, Sickles EA, Mendelson EB, Morris EA. American College of Radiology (ACR) Breast Imaging Reporting and Data System Atlas (BI-RADS Atlas), 5th ed. American College of Radiology 2013.

22. Frank S, Mahdi R, Sherko K. Imaging in patients with breast implants-results of the First International Breast (Implant) Conference 2009. Insights Imaging. 2010;1 (6):93-97.

23. Gutierrez R, Horst KC, Dirbas FM, Ikeda DM. Breast imaging following breast conservation therapy. In: Dirbas FM and Scott-Conner CEH., editor. Breast surgical techniques and interdisciplinary management. New York: Springer Science Business Media; 2011. pp. 975-995.

Source of Support: Nil; Conflict of Interest: None

Submitted: 15-07-2019; Accepted: 24-08-2019; Published online: 19-09-2019 